\title{
An Experimental Study of DNA Rotational Relaxation Time in Nanoslits
}

\author{
Chih-Chen Hsieh, Anthony Balducci, and Patrick S. Doyle* \\ Department of Chemical Engineering, Massachusetts Institute of Technology, 77 Massachusetts Avenue, \\ Cambridge, Massachusetts 02139
}

Received March 8, 2007; Revised Manuscript Received April 30, 2007

\begin{abstract}
The longest relaxation time $\left(\tau_{1}\right)$ of DNA confined in nanoslits is characterized, and its dependence on molecular weight $(M)$ and channel height $(h)$ is investigated. The relaxation time is extracted from the rotational autocorrelation function obtained from time-sequence images of confined DNA at equilibrium using fluorescence microscopy. We find that $\tau_{1} \sim M^{2.45} h^{-0.92}$, in partial agreement with the predictions of the blob theory $\left(\tau_{1} \sim\right.$ $\left.M^{5 / 2} h^{-7 / 6}\right)$. The experimental results suggest that the assumptions of both a 2-dimensional self-avoiding walk of blobs and a 3-dimensional self-avoiding walk of polymer segments within blobs are valid, while the assumption of nondraining blobs is compromised. We also find $\left(\tau_{1} / \tau_{1, \text { bulk }}\right) \sim M^{0.1}\left(R_{\mathrm{g}, \text { bulk }} / h\right)^{0.92}$, where $\tau_{1 \text {,bulk }}$ is the bulk relaxation time and $R_{\mathrm{g}, \text { bulk }}$ the bulk radius of gyration. Because of the very weak $M$ dependence in above scalings, a master plot of $\left(\tau_{1} / \tau_{1, \text { bulk }}\right)$ vs $\left(R_{\mathrm{g} \text {,bulk }} / h\right)$ is constructed and is used to compare our results to other studies. The plot also provides a convenient way to estimate the relaxation time of DNA in varying degrees of confinement. Using the measured relaxation time and blob theory, we explain recent observations that a very large shear rate is required to deform DNA when it is confined to channels with a dimension comparable to or smaller than the bulk radius of gyration.
\end{abstract}

\section{Introduction}

With the development of nanotechnology, new applications have been created for separating and manipulating biomolecules with nanodevices. ${ }^{1-7}$ It is therefore essential to understand the underlying physics and transport properties of highly confined macromolecules. Such knowledge will not only be important for the development of polymer physics but also could help the design and optimization in future applications such as separations and drug delivery.

Slitlike nanochannels with well-controlled dimensions and properties have served as a platform for systematic investigation of the dynamics of confined biomolecules. ${ }^{1,8}$ Such channels can be fabricated with standard microlithography techniques ${ }^{9}$ and can have one dimension small enough to substantially change the behavior of macromolecules. The dynamics of a polymer confined in a slit and a circular tube has been predicted by de Gennes and co-workers ${ }^{10-12}$ using blob theory. The theory describes a confined polymer as a string of blobs with diameters equal to the smallest dimension of the confinement and yields predictions for the scaling of the polymer equilibrium extension, diffusivity, and the longest relaxation time as a function of polymer molecular weight, chain rigidity, and the degree of confinement. In addition to blob theory, Odijk ${ }^{13}$ proposed a deflection chain picture to describe the dynamics of a stiff chain confined in a circular tube with the diameter much smaller than its persistence length, $b$. Although both theories have existed for decades, they have only recently begun to be tested experimentally in well-defined geometries.

Several experimental and simulation studies have investigated the dynamics of DNA confined in rectangular channels. . $^{2,14,15}$ Jendrejack et al. ${ }^{14,15}$ used Brownian dynamics (BD) with a bead-spring model to simulate DNA behavior in a square channel. An excluded volume force was used to mimic the good solvent condition. Both intramolecular and wall-molecule

\footnotetext{
*Corresponding author. E-mail: pdoyle@mit.edu.
}

hydrodynamic interactions were included in the simulations. Although the study focused on shear-induced migration of DNA, the dependence of the equilibrium extension of DNA $\left(\langle x\rangle_{0}\right)$ and the longest relaxation time $\left(\tau_{1}\right)$ on the channel size $(h)$ were also examined. They found that $\langle x\rangle_{0} \sim h^{-2 / 3}$ and $\tau_{1} \sim h^{-1 / 3}$, in agreement with the predictions from blob theory. In an experimental study, Tegenfeldt et al. ${ }^{2}$ showed that DNA were highly extended at equilibrium in nearly square nanochannels, and their equilibrium extensions were found to be proportional to the molecular weight, again in agreement with blob theory. In a later experimental study, Reisner et al. ${ }^{4}$ determined the scaling of the longest relaxation time and equilibrium extension of DNA vs the size of square nanochannels. The longest relaxation times of DNA were extracted from a singleexponential fit to the decay of the autocorrelation function of the DNA extension. A strong transition was observed both in the scalings of $\tau_{1}$ and $\langle x\rangle_{0}$ with $h$ around $h \approx 140 \mathrm{~nm}$, roughly corresponding to twice the persistence length of DNA. The experimental results were compared against the predictions given by blob theory for $h>2 b$ and those inferred from the deflection chain model ${ }^{13}$ for $h<2 b$. However, only a qualitative agreement was reached. Odijk ${ }^{16}$ recently developed a theory to predict DNA extension at equilibrium in circular, square, and rectangular nanochannels. Both the bending free energy that rises from the molecular elasticity and the entropic free energy that rises from the reduction of the orientational and translational degree of freedom of molecule were taken into account in this theory. The predicted DNA stretches were close to those reported by Reisner et al. ${ }^{4}$ and Jo et al. ${ }^{7}$ However, it was noted that the prediction given for DNA extension in a rectangular channel will not be valid for DNA in very wide nanoslits because the assumption of small lateral fluctuations of DNA is not satisfied.

The relaxation behavior of DNA confined in a well-defined slitlike structure was first observed by Bakajin et al. ${ }^{17}$ In this pioneering study, T4-DNA was hooked on obstacles and stretched electrohydrodynamically in small slits with heights of $5 \mu \mathrm{m}, 300 \mathrm{~nm}$, and $90 \mathrm{~nm}$. Their relaxation behavior was 
observed after DNA disengaged from the obstacles and was found to be slower in shallower channels. However, the quantitative values of DNA relaxation time were not provided. Stigter ${ }^{18}$ and Woo et al. ${ }^{19,20}$ performed Brownian dynamics simulations and reported satisfactory results comparing to those of Bakajin et al., but no comparison was made against the prediction of blob theory. Recently, Randall and Doyle ${ }^{21,22}$ and Randall ${ }^{23}$ reported relaxation times of $\lambda$-, $2 \lambda$-, and T4-DNA in $2 \mu \mathrm{m}$ high slits. The relaxation time of confined T4-DNA (with bulk radius of gyration $\left(R_{\mathrm{g}, \text { bulk }}\right)$ of $\sim 1.5 \mu \mathrm{m}$ ) was found to be almost double its bulk value.

The blob theory prediction of the scaling of $\tau_{1}$ of polymers confined in a slit was examined by Hagita et al. ${ }^{24}$ using dynamic Monte Carlo simulation with the bond fluctuation model. They found in good solvent $\tau_{1}$ scales as $M^{2.5}$, and the relaxation spectrum follows the same scaling down to the size of a blob. These findings are in agreement with blob theory. ${ }^{12}$ Although the study did not consider hydrodynamic interactions, the results are still inspiring because blob theory also assumes no hydrodynamic interaction between blobs.

Chen et al. ${ }^{25}$ simulated DNA conformation and diffusion in slits using the method of Jendrejack et al. ${ }^{15}$ The same study also reported experiments of DNA diffusivity in slitlike channels with $0.3<\left(R_{\mathrm{g}, \text { bulk }} / h\right)<1$. The simulations confirmed that the equilibrium DNA stretch is proportional to the $-1 / 4$ power of the slit height as predicted by blob theory. In addition, the simulations and experimental results for DNA diffusivity in different degrees of confinement agreed well with each other and were close to the prediction of blob theory. Usta et al. ${ }^{26}$ employed the lattice Boltzmann method to simulate the confined polymer dynamics. Their results for diffusion of confined polymers in slit channels were essentially the same as those of Chen et al. ${ }^{25}$ However, when we scrutinize the results of Chen et $\mathrm{al}^{25}$ and Usta et al., ${ }^{26}$ it is clear that the dependence of diffusivity on the slit height is weaker than that predicted by blob theory.

Balducci et al. ${ }^{27}$ measured the diffusivity of DNA in nanoslits over a much wider range of conditions $0.4<\left(R_{\mathrm{g} \text {,bulk }} / h\right)<15$. The diffusivity was found to vary inversely with molecular weight when the channel height is smaller than $R_{\mathrm{g} \text {,bulk. This }}$ scaling follows exactly the blob theory prediction and suggests complete screening of hydrodynamic interactions between blobs. A scaling analysis was provided to explain why the algebraic decay of the far-field hydrodynamic interaction is sufficient to eliminate large-length scale hydrodynamic cooperativity in the dynamics of quasi-two-dimensional polymers in good solvents. The scaling of DNA diffusivity with channel height was also investigated in detail. The dependence of DNA diffusivity was weaker than the blob theory prediction. In contrast to Reisner et al., ${ }^{4}$ no drastic change in DNA diffusivity was observed when the height of slits approached the persistence length of DNA.

After surveying previous work, we find the relaxation behavior of a polymer confined in slitlike geometry has not been quantitatively investigated in experiments. The longest relaxation time of a polymer is fundamentally important because it defines the natural time constant of the molecule and characterizes how fast a polymer can react in response to an imposed flow. An important example is that a polymer will not appreciably deform from equilibrium until Weissenberg $\left(W i=\tau_{1} \dot{\gamma}\right)$ approaches $1 .^{28}$ Here $\dot{\gamma}$ is the strain rate and can be either shear rate or elongation rate. Moreover, by determining the dependence of $\tau_{1}$ and $D$ on molecular weight and channel height, we can also investigate the effect of hydrodynamic interactions on the dynamics of a polymer in confinement. The goal of this study is to provide an accurate measurement of the longest relaxation time of DNA confined in slits and to test the applicability of de Gennes' blob theory for this scenario.

\section{Scaling Arguments for Polymer Dynamics in a Slit}

We consider a linear polymer with a persistence length $b$ and a contour length $N b$ confined in a slitlike channel with height $h$ smaller than the natural size of the chain but larger than $b$. Note that $N$ is proportional to $M$, so we treat them as interchangeable variables in scaling expressions. de Gennes ${ }^{10,11}$ has described the behavior of such a chain using the blob picture. In the good solvent limit, de Gennes assumed (1) the chain can be thought of as a string of blobs with each blob having a diameter $h,(2)$ these blobs are confined in the slit so their configuration follows a 2D self-avoiding walk (SAW), (3) there are no hydrodynamic interactions between blobs, and (4) chain segments within blobs are not aware of the presence of the confinement and behave as if they were under a 3D, nonconfined condition. Therefore, segments within a blob will follow a 3D SAW and interact hydrodynamically.

Following the above assumptions, the number of polymer segments $g$ in each blob can be estimated using a simple geometrical argument:

$$
g \sim\left(\frac{h}{b}\right)^{1 / v_{3 \mathrm{~d}}} \simeq h^{5 / 3} b^{-5 / 3}
$$

where $v_{3 \mathrm{~d}}$ is the Flory-Edwards exponent. ${ }^{29}$ Throughout this study, we use " " to indicate that two quantities are proportional to each other, " $\approx$ " to indicate a numerical approximation to the quantity, and " $\simeq$ " to indicate that $v_{2 \mathrm{~d}}$ and $v_{3 \mathrm{~d}}$ have been substituted with their ideal values of $3 / 4$ and $3 / 5,{ }^{10-12,29}$ respectively. Although a more accurate value of $v_{3 \mathrm{~d}}=0.588$ has been obtained using renormalization group theory, ${ }^{30}$ we follow de Gennes and use $v_{3 \mathrm{~d}}=3 / 5$ in all our derivations. We will discuss the influence of using $v_{3 \mathrm{~d}}=0.588$ instead of $v_{3 \mathrm{~d}}=$ $3 / 5$ in a later section. The scaling of the nominal size $R$ of a confined chain can be derived using the assumption of a $2 \mathrm{D}$ SAW of blobs and can be expressed as

$$
R \sim h\left(\frac{N}{g}\right)^{v_{2 \mathrm{~d}}} \sim N^{\nu_{2 \mathrm{~d}}} h^{\left(v_{3 \mathrm{~d}}-v_{2 \mathrm{~d}}\right) / \nu_{3 \mathrm{~d}}} b^{\nu_{2 \mathrm{~d}} / v_{3 \mathrm{~d}}} \simeq N^{3 / 4} h^{-1 / 4} b^{5 / 4}
$$

Equation 2 can also be obtained from Flory theory by minimizing the free energy of a SAW chain confined in a slit. ${ }^{31,32}$ The $N$ and $h$ dependence in eq 2 has been verified by ten Brinke and co-workers using lattice Monte Carlo simulation ${ }^{33}$ and by Chen et al. ${ }^{25}$ using bead-spring Brownian dynamics simulations.

In addition to the static behavior, dynamic properties of a confined chain can also be derived. To obtain the scaling of the longest relaxation time, we consider the force balance on a chain. The elastic force $F_{\text {elastic }}$ required to stretch a polymer from its equilibrium size $R$ to $R+\delta R$ has been shown by de Gennes that ${ }^{12,31,34}$

$$
F_{\text {elastic }}=-\frac{k_{\mathrm{B}} T}{R^{2}} \delta R
$$

where $k_{\mathrm{B}}$ is the Boltzmann constant. Notice the excluded volume effect has been included in $R$. The drag force $F_{\text {drag }}$ applied by the solvent balances the elastic force and can be estimated:

$$
F_{\text {drag }}=\zeta_{\text {chain }} \delta \dot{R}
$$


where $\zeta_{\text {chain }}$ is the drag coefficient of the entire chain. $\delta \dot{R}$ is the time rate of change of $\delta R$. Equating these two forces leads to

$$
\delta \dot{R}=-\frac{k_{\mathrm{B}} T}{\xi_{\text {chain }} R^{2}} \delta R=-\frac{\delta R}{\tau_{1}}
$$

where the longest relaxation time is

$$
\tau_{1}=\frac{\zeta_{\text {chain }} R^{2}}{k_{\mathrm{B}} T}
$$

Since hydrodynamic interaction is assumed to dominate within a blob, the drag coefficient of the chain is described by

$$
\zeta_{\text {chain }} \sim \zeta_{\text {blob }} N_{\text {blob }} \sim h\left(\frac{N}{g}\right) \sim N h^{\left(\nu_{3 \mathrm{~d}}-1\right) / \nu_{3 \mathrm{~d}}} b^{1 / \nu_{3 \mathrm{~d}}} \simeq N h^{-2 / 3} b^{5 / 3}
$$

Substituting eqs 2 and 7 into eq 6 , Brochard ${ }^{12}$ has shown the scaling of the longest relaxation time $\tau_{1}$ vs $N, h$, and $b$ to be

$$
\tau_{1} \sim N^{2 v_{2 \mathrm{~d}}+1} h^{3-\left(2 v_{2 \mathrm{~d}}+1\right) / v_{3 \mathrm{~d}}} b^{\left(2 v_{2 \mathrm{~d}}+1\right) / v_{3 \mathrm{~d}}} \simeq N^{5 / 2} h^{-7 / 6} b^{25 / 6}
$$

The scaling of diffusivity $D$ can be written as

$$
D \sim \frac{k_{\mathrm{B}} T}{\zeta_{\text {chain }}} \sim N^{-1} h^{\left(1-v_{3 \mathrm{~d}}\right) / v_{3 \mathrm{~d}}} b^{-1 / v_{3 \mathrm{~d}}} \simeq N^{-1} h^{2 / 3} b^{-5 / 3}
$$

This scaling of $D$ with $N$ has been confirmed in our previous study. ${ }^{27}$ However, the measured $D$ showed a weaker dependence on $h$ than predicted by eq 9 .

We now consider another extreme case where the hydrodynamic interactions vanish even within blobs. The overall drag coefficient of a chain is then

$$
\xi_{\text {chain }} \sim \zeta_{\mathrm{b}} N \sim b N
$$

where $\zeta_{\mathrm{b}}$ is the drag coefficient of a single segment. While $\zeta_{\mathrm{b}}$ should be a function of $h$, we treat it as a constant because the typical width of a molecule (about $2 \mathrm{~nm}$ for a double-strained DNA) is much smaller than the slit heights considered in this study. ${ }^{35}$ Using the same analysis as we have used to obtain eqs 8 and 9 , we reach

$$
\begin{gathered}
\tau_{1} \sim N^{2 v_{2 \mathrm{~d}}+1} h^{\left(2 v_{3 \mathrm{~d}}-2 v_{2 \mathrm{~d}}\right) / v_{3 \mathrm{~d}}} b^{2 v_{2 \mathrm{~d}} / v_{3 \mathrm{~d}}+1} \simeq N^{5 / 2} h^{-1 / 2} b^{5 / 2} \\
D \sim N^{-1} b^{-1}
\end{gathered}
$$

The $N$ and $h$ dependence of eq 11 has been verified by Milchev and Binder ${ }^{36}$ and Hagita et al. ${ }^{24}$ using bond fluctuation Monte Carlo simulations. For convenience, we will refer to eqs 8 and 9 as Zimm-blob scaling and eqs 11 and 12 as Rouse-blob scaling.

Comparing experimental results with the predicted scalings, we should be able to verify the assumptions made in blob theory. For example, using the experimental scaling of $D \sim N^{-1}$ alone, one can readily infer that hydrodynamic interaction is screened between blobs. ${ }^{27}$ However, it is not as straightforward to verify other assumptions because the dependence of $D$ and $\tau_{1}$ with $N$ and $h$ is usually a convolution of several assumptions. Therefore, we rearrange the above equations so individual assumptions can be independently examined. We find that although both $D$ and $\tau_{1}$ depend on $\zeta_{\text {chain }}$, their product is independent of $\zeta_{\text {chain }}$ and is only a function of $R$ :

$$
D \tau_{1} \sim R^{2} \sim N^{2 v_{2 \mathrm{~d}}} h^{2\left(v_{3 \mathrm{~d}}-v_{2 \mathrm{~d}}\right) / v_{3 \mathrm{~d}}} \simeq N^{3 / 2} h^{-1 / 2}
$$

Therefore, we can calculate the experimental value of $v_{2 \mathrm{~d}}\left(v_{2 \mathrm{~d} \text { exp }}\right)$ from the scaling of $D \tau_{1}$ with $N$ and subsequently estimate the experimental value of $v_{3 \mathrm{~d}}\left(\nu_{3 \mathrm{~d} \text {,exp }}\right)$ from the scaling of $D \tau_{1}$ with $h$. Once $v_{2 \mathrm{~d} \text {,exp }}$ and $v_{3 \mathrm{~d} \text {,exp }}$ are known, we can substitute them back to eqs $8,9,11$, and 12 to infer the expected dependence of $\tau_{1}$ and $D$ on $h$ and to verify the assumption of dominating hydrodynamic interactions in blobs.

\section{Longest Relaxation Time for Polymer Molecules}

It is worthwhile to briefly review the definition of the longest relaxation time of a polymer chain in dilute solutions. For a polymer chain in either theta or good solvent, both Rouse and Zimm models predict that the longest conformational relaxation time $\left(\tau_{1, \mathrm{c}}\right)$ and the longest rotational relaxation time $\left(\tau_{1, \mathrm{r}}\right)$ are equal, while the longest stress relaxation time $\left(\tau_{1, \mathrm{~s}}\right)$ is half of $\tau_{1, \mathrm{c}} \cdot{ }^{30,32}$ Here $\tau_{1, \mathrm{c}}$ can be defined by the ratio of the drag coefficient to the spring constant of the first normal coordinate. $\tau_{1, \mathrm{r}}$ can be defined by the inverse of the dominating exponential decay rate of the autocorrelation function of the end-to-end vector of a chain. In single-molecule experiments using fluorescence microscopy, $\tau_{1, \mathrm{~s}}$ of DNA in bulk solutions is typically obtained by fitting the decay of the mean-squared stretch of an initially extended chain to a single exponential in the linear regime. ${ }^{21,22,37-39}$ This method has been widely used because it requires relatively small ensembles due to the large perturbation from equilibrium. On the other hand, $\tau_{1, \mathrm{c}}$ and $\tau_{1, \mathrm{r}}$ are difficult to measure in bulk solutions because one needs to collect data over larger ensembles of many relaxation times to extract the time constant, but a molecule can stay in focus only for a short period of time. However, the rotational relaxation time of so-called type A polymer that carries a molecular dipole parallel to its end-to-end vector can be measured by dielectric spectroscopy. ${ }^{40}$ In most single-molecule studies, $\tau_{1, \mathrm{~s}}$ is implicitly designated as the longest relaxation time. Therefore, we follow this tradition in the current study to avoid possible ambiguity.

\section{Experiments}

4.1. Channel and DNA Preparation. The nanoslits used in this study were made with glass using the method described in Mao and Han. ${ }^{9}$ The slits are $150 \mu \mathrm{m}$ wide and $92-760 \mathrm{~nm}$ high. Their heights were determined from SEM images. The DNA samples used are $\lambda$-DNA halfmers $(1 / 2 \lambda, 24.25 \pm 0.25 \mathrm{kbp}), \lambda$-DNA $(\lambda$, $48.5 \mathrm{kbp}), \lambda$-DNA dimers ( $2 \lambda, 97 \mathrm{kbp})$, T4GT7-DNA (T4, 165.6 $\mathrm{kbp}$ ), and a bacterial artificial chromosome (BAC) clone (12M9) (185 kbp). $\lambda$-DNA was purchased from New England Biolabs (NEB). $\lambda$-DNA halfmers were made by digesting $\lambda$-DNA with $\mathrm{XbaI}$ restriction enzyme(NEB). $\lambda$-DNA dimers were made using an oligoprotected ligation. ${ }^{41}$ T4GT7-DNA was purchased from Nippon Gene. BAC-DNA was donated by U.S. Genomics. DNA samples were stained with YOYO-1 fluorescent dye (Molecular Probes) overnight at room temperature at a concentration of 1 dye molecule every 4 base pairs. DNA samples were diluted 2-10-fold immediately before experiments to reach an optimal concentration for observation. The experimental buffer is $0.5 \mathrm{X}$ TBE (Tris-BorateEDTA) solution with an oxygen scavenger system consisting of $4 \% \beta$-mercaptoethanol (CabioChem), $7.26 \mathrm{mg} / \mathrm{mL} \beta$-D glucose, $0.37 \mu \mathrm{L} / \mathrm{mL}$ catalase (Roche), and $0.16 \mathrm{mg} / \mathrm{mL}$ glucose oxidase (Roche). The buffer viscosity $\eta_{\mathrm{s}}$ is $1.075 \mathrm{cP}$ at $22^{\circ} \mathrm{C}, 13.3 \%$ higher than water viscosity measured at the same temperature. The viscosity was determined using microrheology by measuring the diffusivity of $0.925 \mu \mathrm{m}$ polystyrene beads (Polysciences). Nanochannels were filled the night before experiments with $0.5 \mathrm{X}$ TBE buffer and flushed by an electroosmosis flow at a field of $10 \mathrm{~V} / \mathrm{cm}$ to remove bubbles and any residual chemicals left from the fabrication 
process. The flushing buffer was replaced with the experimental buffer $2 \mathrm{~h}$ before experiments. A low electric field was used to prevent ion polarization in the channel. ${ }^{42}$ DNA were driven into the channel using a field of $\sim 30 \mathrm{~V} / \mathrm{cm}$.

4.2. Image Acquisition and Analysis. We observed single DNA molecules using an inverted Zeiss Axiovert 200 microscope with a Plan-Apochromat 100X (N.A. 1.40) oil-immersed objective. The images were taken with a Hamamatsu EB-CCD camera (model 7190-21) at the rate of 30 frames/s for all experiments except for T4-DNA and BAC-DNA (15 frames/s due to their long relaxation times). With our setup, 1 pixel represents $0.135 \mu \mathrm{m}$. 3600 frames were collected for each molecule. Averages were taken over ensembles containing 20-35 molecules. Images were processed using custom-developed code in Interactive Data Language (IDL). The background signal was subtracted from the image before the analysis. With the assumptions that the dyes are evenly distributed along the backbone of a DNA and the camera gain is a linear function of the fluorescent signal, the fluorescent intensity in a pixel is proportional to the total mass of DNA in that pixel. We calculate the total fluorescent intensity $\left(I_{0}\right)$, the center of the mass vector $\left(\mathbf{r}_{\mathrm{cm}}\right)$, and the radius of gyration tensor $(\mathbf{G})$ of a DNA molecule in each frame:

$$
\begin{gathered}
I_{0}(t)=\sum_{m, n} I_{m n}(t) \\
r_{\mathrm{cm}, i}(t)=\frac{1}{I_{0}(t)} \sum_{m, n} r_{m n, i}(t) I_{m n}(t) \\
G_{i j}(t)=\frac{1}{I_{0}(t)} \sum_{m, n}\left(r_{m n, i}(t)-r_{\mathrm{cm}, i}(t)\right)\left(r_{m n, j}(t)-r_{\mathrm{cm}, j}(t)\right) I_{m n}(t)
\end{gathered}
$$

where $I_{m n}$ is the fluorescent intensity of the pixel $[m, n]$, and $i$ and $j$ represent $x$ or $y$ directions that are perpendicular to the smallest dimension of the slit ( $z$ direction). The in-plane diffusivity $(D)$ can be obtained from the mean-square-displacement (MSD): ${ }^{43}$

$$
\begin{array}{r}
\mathrm{MSD}=\left\langle\left[r_{\mathrm{cm}, x}(t)-r_{\mathrm{cm}, x}(t-\delta t)\right]^{2}+\left[r_{\mathrm{cm}, y}(t)-r_{\mathrm{cm}, y}(t-\delta t)\right]^{2}\right\rangle= \\
4 D \delta t(17)
\end{array}
$$

where $\delta t$ is the lag time.

The radius of gyration tensor $(\mathbf{G})$ carries information about the instantaneous size, shape, and orientation of the DNA. The radius of gyration $\left(R_{\mathrm{g}}\right)$ is the square root of the trace of $\mathbf{G}$. The eigenvectors of $\mathbf{G}$ represent the principal and minor axes that describe the temporal orientation of the DNA. More importantly, the longest relaxation time of the DNA can be extracted from the rate of the change of DNA orientation. ${ }^{44,45}$ We first calculate the angle between the principal eigenvector and the $x$-axis:

$$
\theta(t)=\arctan \left(\frac{\lambda(t)-G_{x x}(t)}{G_{x y}(t)}\right), \quad \frac{\pi}{2}>\theta(t)>-\frac{\pi}{2}
$$

where $\lambda(t)$ is the eigenvalue associated with the principal eigenvector. The time autocorrelation function of $\theta(t)$ is defined by 45

$$
C_{\mathrm{r}}(\delta t)=\frac{\left\langle\left(\theta(t)-\theta_{0}\right)\left(\theta(t-\delta t)-\theta_{0}\right)\right\rangle}{\left\langle\left(\theta(t)-\theta_{0}\right)^{2}\right\rangle} \sim \exp \left(-\delta t / \tau_{1}\right) \quad \text { for } \delta t \geq \tau_{1}
$$

where \langle\rangle denotes the ensemble average. $\theta_{0}$ is the equilibrium average of $\theta(t)$ and is taken to be zero in our calculation. ${ }^{46} \mathrm{We}$ will show that this assumption is valid in a later section.

The physical reason that one can obtain the longest relaxation time by tracking the rotation of the principal eigenvector $(\mathbf{P})$ of the radius of gyration tensor is because $\mathbf{P}$ is always parallel to the first normal coordinate of a chain ${ }^{47}$ that represents the slowest mode of the chain's internal motion. We emphasize that the rotational relaxation time obtained by our method will be equal to $\tau_{1, \mathrm{~s}}$ or half

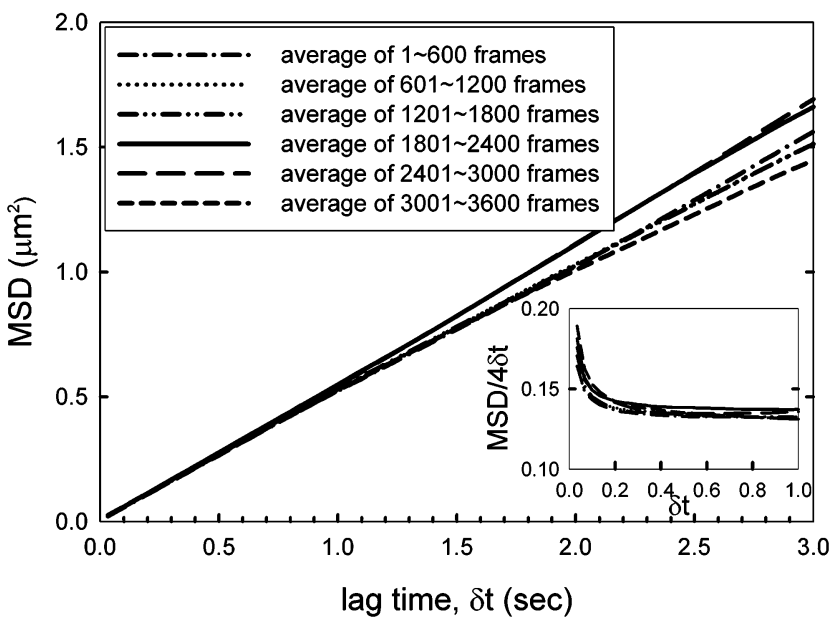

Figure 1. Analysis of $\lambda$-DNA diffusion in a $223 \mathrm{~nm}$ high slit from different time segments of the experiment movies. The mean-squaredisplacement curves fluctuate at long lag times $(\delta t>1 \mathrm{~s})$ due to limited statistics, but the deviation among the ensemble is not systemic. The inserted plot shows MSD/4 $\delta t\left(\mu \mathrm{m}^{2} / \mathrm{s}\right)$ vs $\delta t$. Despite the initial surge caused by the noise in the DNA images, the value of MSD/4 $\delta t$ is fairly constant for $0.2<\delta t<1 \mathrm{~s}$. The frame rate for this data is 30 frames/s.

of the true rotational relaxation time $\tau_{1, \mathrm{r}}$ because the orientation of the principal axis is defined between $\pi / 2$ and $-\pi / 2$. Since the phase space is cut to half, the measured relaxation time becomes half of the true $\tau_{1, \mathrm{r}}$. This fact has been verified using BD simulations.

A small amount of photobleaching will always occur in single molecule DNA studies. We must carefully examine our data to make sure the DNA physical properties are temporally invariant. Two detrimental effects may associate with photobleaching on a stained DNA. Its contour length may shorten with time, and it may be nicked and eventually fragment. ${ }^{41}$ We expect that DNA will diffuse and relax faster if it becomes shorter or more flexible. Therefore, by checking the time variation during the course of an experiment of either DNA diffusivity or relaxation time, we should be able to determine whether DNA is intact through the experiments. Since we are not able to obtain accurate relaxation times using short movies, we choose to use diffusivity as the indicator. We cut each of our movies into six submovies and compare the MSD curves from the ensemble average of the submovies. Figure 1 shows the result of such an analysis for $\lambda$-DNA in a $223 \mathrm{~nm}$ high slit. The MSD curves of the six different ensembles are very close to each other for $\delta t<1 \mathrm{~s}$. They fluctuate at long lag time space $(\delta t>1 \mathrm{~s})$ due to limited statistics while no sign of systemic change is observed. The inserted plot shows the value of MSD/4 $\delta t$ for $\delta t<1$. The curve starts high and decays to a constant after $0.2 \mathrm{~s}$. This initial surge is mainly caused by the noise in the DNA images. The same phenomenon occurs in colloid particle tracking experiments and has been investigated in detail by Savin and Doyle. ${ }^{43}$ After the initial $0.2 \mathrm{~s}$, the value of MSD/4 $\delta t$ is fairly constant and does not systematically vary over the six ensembles.

\section{Results and Discussion}

5.1. Measuring DNA Relaxation Time from Rotational Autocorrelation Function. Given time-sequence images of DNA, one can extract the relaxation time not only from the rotational autocorrelation function but also from the stretch autocorrelation function $\left(C_{\mathrm{s}}(\delta t)\right)$ that can be defined in a similar way as eq 19 with $\theta$ replaced with DNA stretch. However, we choose to measure $\tau_{1}$ from the rotational autocorrelation function for two main reasons. First, the relaxation time measured from the stretch autocorrelation function is greatly affected by the blooming effect that also decays with time due to the photobleaching of fluorescent dyes. Therefore, $C_{\mathrm{s}}(\delta t)$ becomes a 
(a)
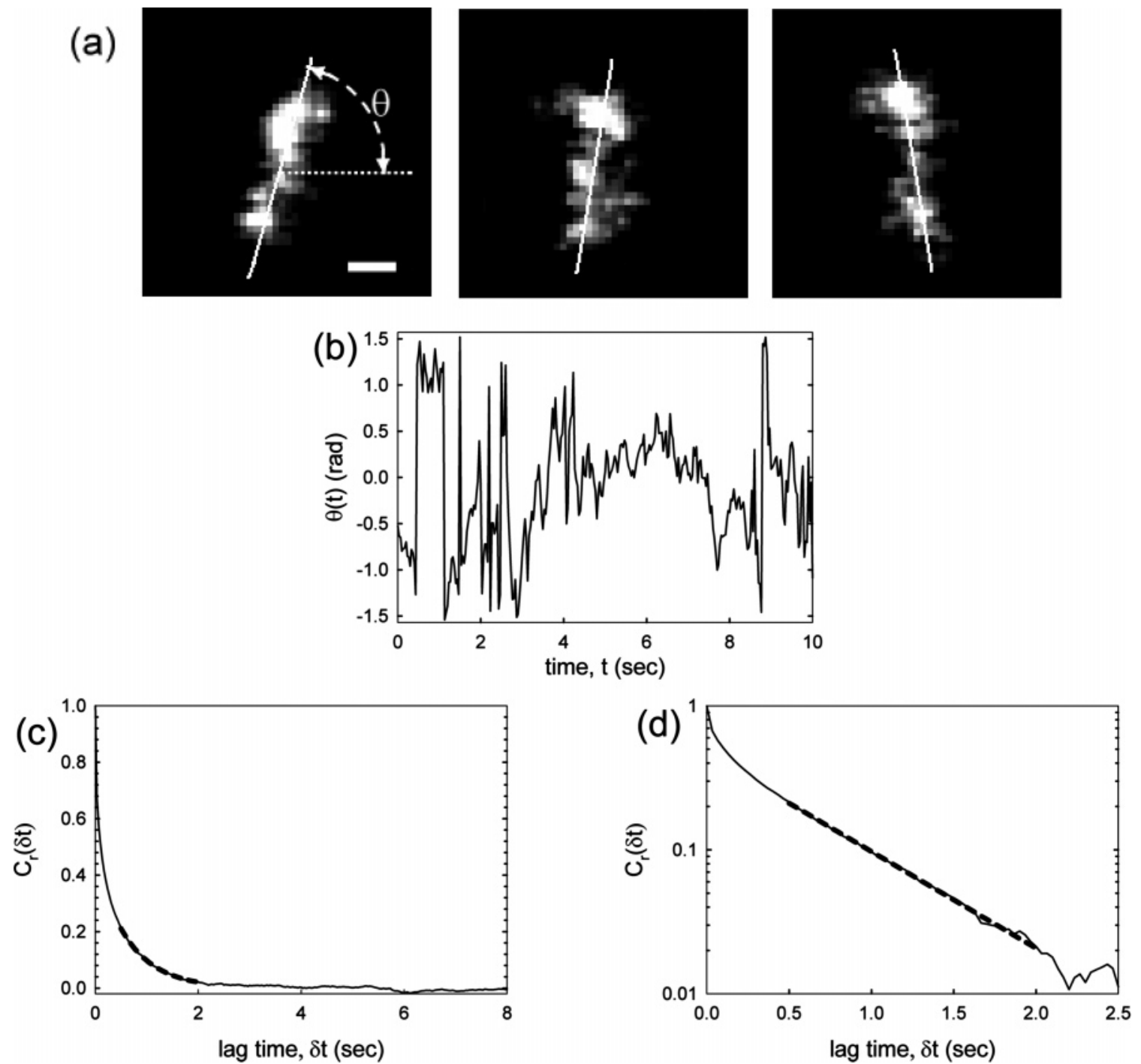

Figure 2. (a) A time sequence of a $\lambda$-DNA confined in a $223 \mathrm{~nm}$ nanoslit. The time interval between each image is $0.1 \mathrm{~s}$. The solid line overlaying the DNA is the direction of the principal axis of the DNA calculated from the radius of gyration tensor. The orientation angle of the principal axis is designated as $\theta$. The scale bar in the first image is $1 \mu \mathrm{m}$. (b) A typical time evolution of $\theta$. (c) The ensemble average of the time autocorrelation functions of $\theta$ vs $\delta t$ in a linear scale. The solid line is the experimental result, and the dashed line is a single-exponential fit to extract the longest relaxation time from the data. This set of data is also measured from $\lambda$-DNA in a $223 \mathrm{~nm}$ slit, and the longest relaxation time is found to be $0.67 \mathrm{~s}$. (d) The same plot as (c) but in a semilog scale.

convolution of two decay modes. When this happens, $C_{\mathrm{s}}(\delta t)$ does not become a single-exponential decay even for $\delta t \geq \tau_{1}$. Although we have tried to remove this artifact by making a correction to the raw data, the resulting autocorrelation curves are not consistently as good as those from the rotational autocorrelation function. On the other hand, since the blooming effect is isotropic, it has minimum effect on the measured orientation of the DNA.

Second, it is difficult to estimate accurately the average stretch of DNA. Since the average value of the stretch must be subtracted from the temporal stretch in the calculation of $C_{\mathrm{s}}(\delta t)$, its accuracy greatly affects the measured $\tau_{1}$. We have characterized such effects using Brownian dynamics simulations and found that errors in the average stretch generally lead to a smaller relaxation time compared to the true relaxation time. The signature of this problem is that the autocorrelation function does not decay to zero in the long time limit. In contrast, $\theta_{0}$ requires no estimation from the experiments and should be zero because the average orientation of a DNA is isotropic. We have checked this assumption in our experiments and found it to be valid.
Figure $2 \mathrm{a}$ shows a time sequence of a $\lambda$-DNA confined in a $223 \mathrm{~nm}$ slit, and Figure 2b shows a typical time evolution of the orientation angle $\theta(t)$ of a $\lambda$-DNA in this condition. Figure $2 \mathrm{c}$ shows the rotational autocorrelation function vs lag time in a linear scale. The dashed line is the single-exponential fit to extract the longest relaxation time. This plot also demonstrates that $C_{\mathrm{r}}(\delta t)$ decays to zero in the long lag time limit and confirms the validity of our assumption of $\theta_{0}=0$. Figure $2 d$ is identical to Figure 2c, but in a semilog scale. As can be seen, the autocorrelation function becomes a single-exponential decay when the lag time $\delta t$ approaches $\tau_{1} \approx 0.67 \mathrm{~s}$.

5.2. Relaxation Time and Diffusivity Scalings vs Channel Height. We have measured the diffusivity and the longest relaxation time of $\lambda$-DNA in nanoslits with heights of 760,475 , 223 , and $92 \mathrm{~nm}$. The radius of gyration of $\lambda$-DNA is $\sim 670 \mathrm{~nm}$ using the relation $R_{\mathrm{g}}=0.203\left(k_{\mathrm{B}} T / \sqrt{6} \eta_{\mathrm{s}} D_{\text {bulk }}\right)^{30}$ and measured value $D_{\text {bulk }}=0.47 \pm 0.03 \mu \mathrm{m}^{2} / \mathrm{s}$. The results of DNA diffusivity vs channel height are shown in Figure 3a. We also plot the blob theory scalings with both Zimm-blob (eq 9) and Rouseblob (eq 12) assumptions. As can be seen, the diffusivity of 

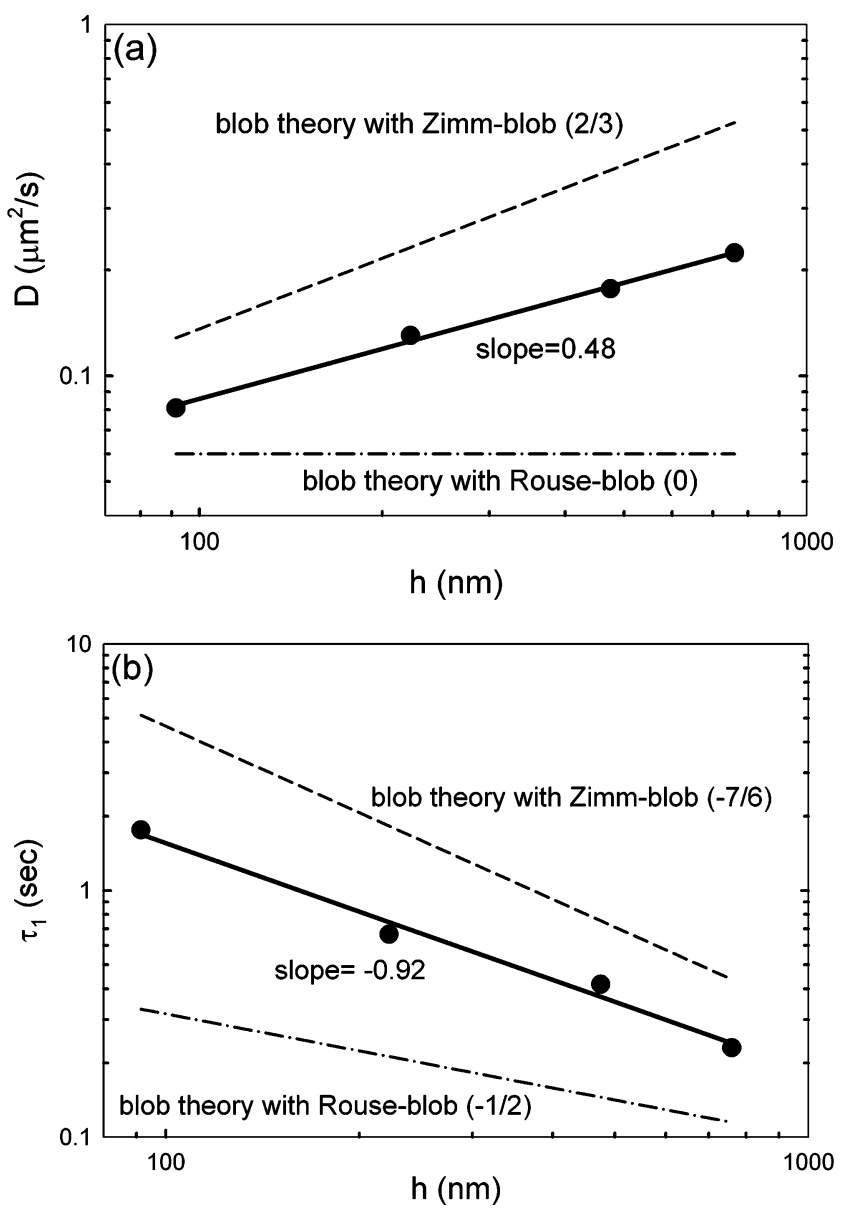

Figure 3. (a) Diffusivity vs channel height for $\lambda$-DNA. The slope of the best-fitting curve through the data is $0.48 \pm 0.03$. The slope of the Zimm-blob scaling (dashed line) is $2 / 3$, while the slope of the Rouseblob scaling (dash-dot line) is 0 . (b) The longest relaxation time vs the channel height for $\lambda$-DNA. The slope of the best-fitting curve through the experimental data is $-0.92 \pm 0.08$. The slope of the Zimm-blob scaling is $-7 / 6$, and slope of the Rouse-blob scaling is -0.5 .

$\lambda$-DNA decreases with the decreasing channel height. The slope of the regression curve is $0.48 \pm 0.03$. This scaling is between the Zimm-blob and Rouse-blob scalings and may suggest the partial-draining nature of the blobs in our experiments.

We also compare the DNA diffusivity with those measured in our previous study. ${ }^{27}$ Although the fitted slope of $D$ vs $h$ in the current study is slightly lower than that in the previous study $^{27}(0.55 \pm 0.05)$ over a similar range of channel heights, they are still within the error range. However, the absolute values of DNA diffusivity are constantly higher than those reported in our previous work by about $10 \%$. The experimental setup of the current study is identical to that of our previous study except that an extra oxygen scavenger system consisting of $\beta$-D-glucose, catalase, and glucose oxidase is introduced to the experimental buffer to further suppress photobleaching and to extend the lifetime of DNA in experiments. Similar buffers have been used by the Chu group. ${ }^{38,48} \mathrm{We}$ call the buffers used in the previous and the current studies as BME and GLOX buffers, respectively. A $\lambda$-DNA molecule can only be continuously observed with our experimental setup for less than $30 \mathrm{~s}$ without breaking in BME buffer, while it can stay intact for at least few minutes in GLOX buffer. This prolonged observation time allows us to measure the DNA relaxation time more accurately. Moreover, we cannot access the long relaxation time of BAC-DNA without the GLOX buffer.
Table 1. Comparison of Buffer Viscosity and Confined $\lambda$-DNA Diffusivity Measured in BME and GLOX Buffers (Temperature $22-23{ }^{\circ} \mathrm{C}$ )

\begin{tabular}{|c|c|c|}
\hline buffer & BME buffer & GLOX buffer \\
\hline buffer viscosity (cP) & 1.05 & 1.075 \\
\hline $\begin{array}{l}\lambda \text {-DNA diffusivity in bulk solution } \\
\left(\mu \mathrm{m}^{2} / \mathrm{s}\right)\end{array}$ & $0.456 \pm 0.041$ & $0.474 \pm 0.032$ \\
\hline $\begin{array}{l}\lambda \text {-DNA diffusivity in } 223 \mathrm{~nm} \text { slit } \\
\left(\mu \mathrm{m}^{2} / \mathrm{s}\right)\end{array}$ & $0.118 \pm 0.004$ & $0.130 \pm 0.002$ \\
\hline $\begin{array}{l}\lambda \text {-DNA diffusivity in } 92 \mathrm{~nm} \text { slit } \\
\left(\mu \mathrm{m}^{2} / \mathrm{s}\right)\end{array}$ & $0.071 \pm 0.003$ & $0.081 \pm 0.001$ \\
\hline
\end{tabular}

To make sure the discrepancy in the measured DNA diffusivity is not caused by the channels or due to human error, we redo the experiments using $\lambda$-DNA with the BME and GLOX buffers in 92 and $223 \mathrm{~nm}$ slits and confirm that the difference in $\lambda$-DNA diffusivity still exists. Table 1 summarizes the buffer viscosities and $\lambda$-DNA diffusivity in 92 and $223 \mathrm{~nm}$ slits. Since everything else is identical between the current and our previous studies, we suspect that the agents of the oxygen scavenger system may have slightly changed either the solvent quality or the persistence length of the DNA. We briefly check these two factors independently. If solvent quality becomes worse, a DNA coil shrinks and diffuses faster. However, blob theory predicts that if the solvent quality becomes worse and thus $v_{3 \mathrm{~d}}$ becomes smaller, the diffusion coefficient should become more strongly dependent on the channel height. Such effect is not seen in our experiments because the diffusion coefficient becomes more weakly dependent on the channel height once GLOX buffer is used (see Figure 3a). On the other hand, we notice that the diffusivity of DNA is proportional to $b^{-2 / 5}$ in bulk solutions, ${ }^{30}$ while blob theory predicts $D \sim b^{-2 / 3}$ in a slit. Thus, a $13 \%$ reduction in the persistence length will result in an increase of DNA diffusivity for $6 \%$ in bulk solutions and $10 \%$ in confinement. This picture is consistent with our results (see Table 1); however, we cannot fully confirm our explanation due to the magnitude of the error associated with the measured bulk diffusivity.

The relaxation time vs channel height is shown in Figure 3b. We also plot blob theory scalings with both Zimm-blob (eq 8) and Rouse-blob (eq 11) assumptions. The experimental relaxation time follows the slope of $-0.92 \pm 0.08$ that again falls between the predicted scalings with Zimm-blob (-7/6) and Rouse-blob $(-1 / 2)$ but is closer to Zimm scaling. With the above results, it is tempting to conclude that the blobs are partialdraining. However, we notice that the scalings of $D$ and $\tau_{1}$ vs $h$ also depend on the values of $v_{2 \mathrm{~d}}$ and $v_{3 \mathrm{~d}}$ (see eqs $8,9,11$, and 12). Therefore, we cannot draw the conclusion before $v_{2 \mathrm{~d}}$ and $v_{3 \mathrm{~d}}$ are independently verified.

5.3. Relaxation Time and Diffusivity Scalings vs DNA Molecular Weight. The relaxation time and the diffusivity of DNA with different molecular weights are measured in $475 \mathrm{~nm}$ slits. The DNA diffusivity is found to be inversely proportional to its molecular weight (see Figure 4a). This result agrees with our previous study and with the predictions given by both eq 9 and eq 12. It indicates that hydrodynamic interactions are totally screened between blobs but provides no information about hydrodynamic interactions within blobs. Figure $4 \mathrm{~b}$ compares DNA relaxation times vs their molecular weights relative to the molecular weight of $\lambda$-DNA. The slope of the best fitted line over the experimental data is $2.45 \pm 0.04$, very close to the theoretical value 2.5 predicted by eqs 8 and 11 . As discussed in an earlier section, this 2.5 power prediction arises from the assumptions of 2D SAW of blobs and absence of hydrodynamic interaction between blobs. Since the latter assumption has been 

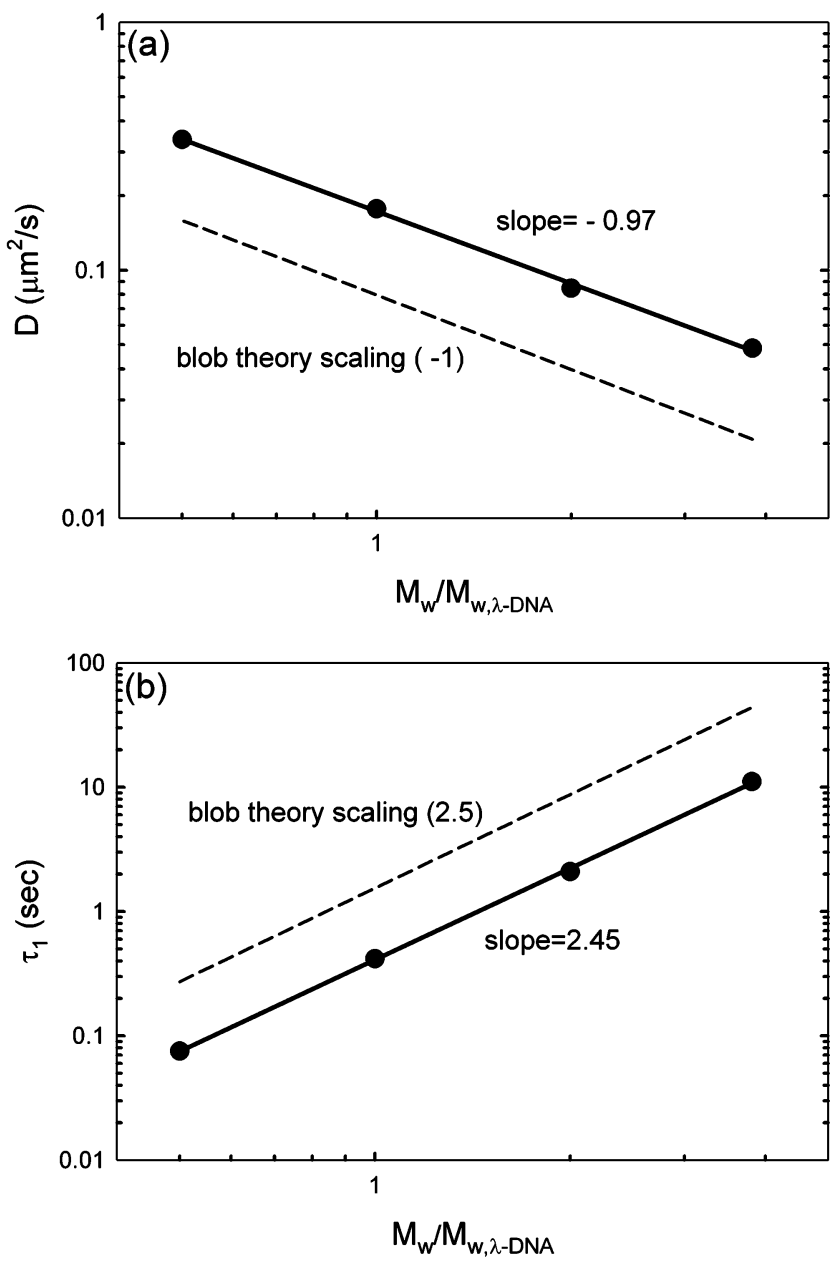

Figure 4. (a) Diffusivity vs molecular weight $\left(M_{\mathrm{w}}\right)$ relative to that of $\lambda$-DNA $\left(M_{\mathrm{w}, \lambda \text {-DNA }}\right)$ in $475 \mathrm{~nm}$ slits. The slope of the best-fitting curve through the experimental data is $-0.97 \pm 0.03$. The slope of the dashed line is -1 that is predicted in both eq 9 and eq 12. (b) The longest relaxation time vs molecular weight $\left(M_{\mathrm{w}}\right)$ relative to that of $\lambda$-DNA in $475 \mathrm{~nm}$ slits. The slope of the best-fitting curve through the experimental data is $2.45 \pm 0.04$, in agreement with the blob theory scaling (2.5).

confirmed, we can infer that DNA blobs in our system confined in a thin slit do follow a 2D SAW.

5.4. Verification of Assumptions Made in Blob Theory. As we have pointed out in earlier sections, it is important to verify the values of $v_{2 \mathrm{~d}}$ and $v_{3 \mathrm{~d}}$ in order to fully test de Gennes' assumptions, and this can be achieved by examining the scalings of $D \tau_{1}$ with $N$ and $h$, respectively. As can be seen in Figure 5a,b, $D \tau_{1} \sim N^{1.48 \pm 0.06} h^{-0.45 \pm 0.06}$. Comparing eq 13 with the experimental scaling $D \tau_{1} \sim N^{1.48}$, we can calculate $v_{2 \mathrm{~d} \text {,exp }}$ to be 0.74 (0.739). Then, substituting $v_{2 \text { d,exp }}$ to eq 13 and comparing it with $D \tau_{1} \sim h^{-0.45}$, we obtain $v_{3 \text { d,exp }}$ to be $0.60(0.603)$. The numbers in ( ) are the values of $v_{2 \mathrm{~d} \text { exp }}$ and $v_{3 \mathrm{~d} \text {,exp }}$ to three significant figures to highlight the small deviation in $v_{2 \mathrm{~d} \text {,exp }}$ and $\nu_{3 \mathrm{~d} \text {,exp }}$ from their ideal values. Since $D \tau_{1}$ is only a function of $R$, the estimated values of $v_{2 d \text {,exp }}$ and $v_{3 d \text {,exp }}$ are free from the influence of the drag coefficient which contains the information on hydrodynamic interactions within blobs. Giving the error associated with our experiments, $v_{2 \mathrm{~d} \text {,exp }}$ and $v_{3 \mathrm{~d} \text {,exp }}$ are indistinguishable from $v_{2 \mathrm{~d}}=3 / 4$ and $v_{3 \mathrm{~d}}=3 / 5$ used by de Gennes. Therefore, the assumptions of 2D SAW of blobs and 3D SAW of segments within blobs are valid.

Substituting $v_{2 \mathrm{~d} \text {,exp }}$ and $v_{3 \mathrm{~d} \text {,exp }}$ into eqs $8,11,9$, and 12 , we obtain the expected scalings that have accounted for the slight deviation in $v_{2 \mathrm{~d}}$ and $v_{3 \mathrm{~d}}$ observed experimentally:
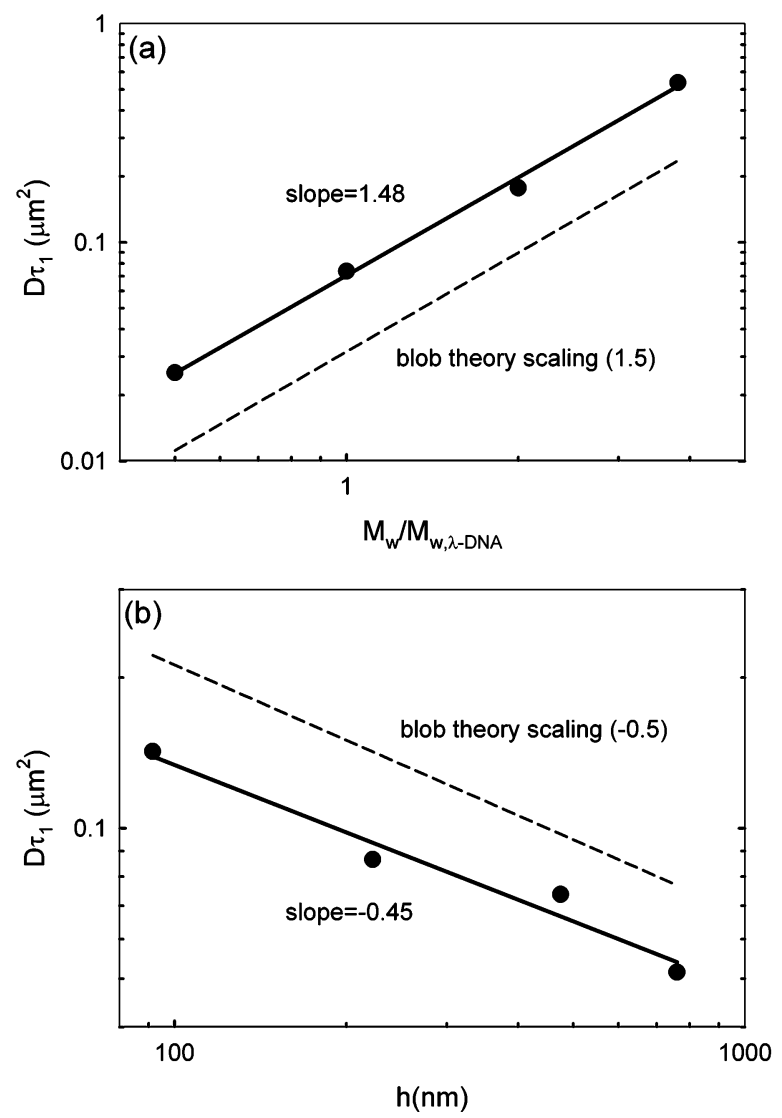

Figure 5. (a) Product of diffusivity and the longest relaxation time vs molecular weight relative to that of $\lambda$-DNA in $475 \mathrm{~nm}$ slits. The slope of the best-fitting curve is $1.48 \pm 0.06$. The slope of the dashed line is 1.5 , predicted by eq 13 using $v_{2 \mathrm{~d}}=(3 / 4)$. (b) Product of diffusivity and the longest relaxation time vs channel height for $\lambda$-DNA. The slope of the best-fitting curve of experimental data is $-0.45 \pm 0.06$. The dashed line with the slope of -0.5 represents the scaling predicted by eq 13 using $v_{2 \mathrm{~d}}=3 / 4$ and $v_{3 \mathrm{~d}}=3 / 5$.

$$
\begin{gathered}
\tau_{1, \text { Zimm-blob }} \sim N^{2.48} h^{-1.11} \\
\tau_{1, \text { Rouse-blob }} \sim N^{2.48} h^{-0.45} \\
D_{\text {Zimm-blob }} \sim N^{-1} h^{0.66} \\
D_{\text {Rouse-blob }} \sim N^{-1}
\end{gathered}
$$

Comparing these scalings with experimental results in Figure 3 , we can readily confirm that blobs are partial-draining, not nondraining as de Gennes has assumed. It is also worthwhile to note that the experimental scaling of $D \tau_{1}$ with $h$ seems to deviate considerably from de Gennes' prediction, which leads one to expect a sizable discrepancy between $v_{3 \mathrm{~d} \text {,exp }}$ and $v_{3 \mathrm{~d}}$. However, $v_{3 \mathrm{~d} \text {,exp }}$ is found almost identical to $v_{3 \mathrm{~d}}$. The discrepancy in the scaling of $D \tau_{1}$ with $h$ is found to be caused by the combined effect of the slight deviations in $v_{2 \text { d,exp }}$ and $v_{3 \mathrm{~d} \text {,exp }}$ from their ideal values. It highlights the significance of our argument that one might be mislead to erroneous conclusions by comparing the experimental data with the blob theory scalings without noticing the convolution of several assumptions.

As we have mentioned in an earlier section, the more accurate value of $v_{3 \mathrm{~d}}$ is 0.588 . Therefore, we want to clear any concern that using $v_{3 \mathrm{~d}}=0.588$ may affect our conclusion. Substituting $v_{2 \mathrm{~d}}=3 / 4$ and $v_{3 \mathrm{~d}}=0.588$ into eqs $8,11,9$, and 12 , we obtain $\tau_{1, \text { Zimm-blob }} \sim N^{2.5} h^{-1.25}, \tau_{1, \text { Rouse-blob }} \sim N^{2.5} h^{-0.55}, D_{\text {Zimm-blob }} \sim$ $N^{-1} h^{0.7}$, and $D_{\text {Rouse-blob }} \sim N^{-1}$. We note that the scalings of $\tau_{1}$ 


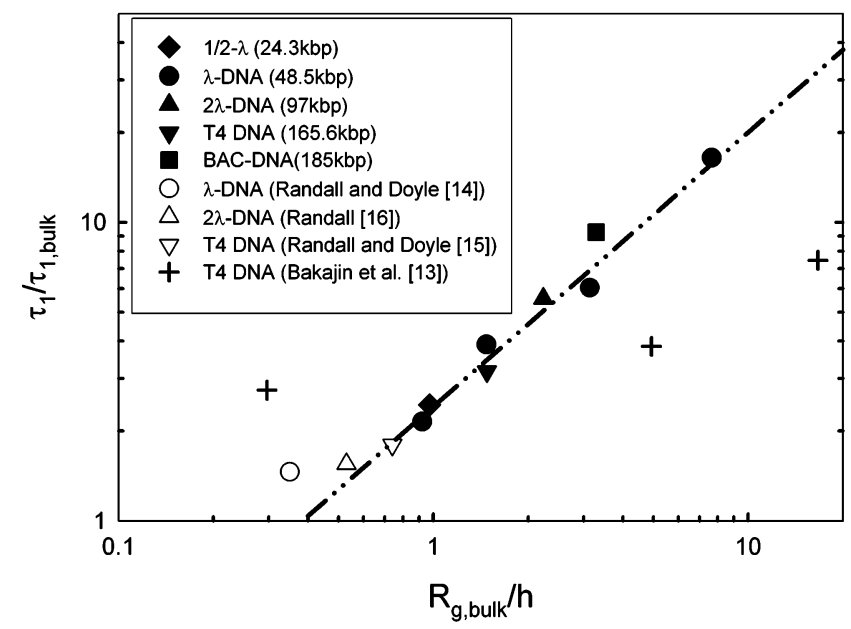

Figure 6. A master plot of the longest relaxation time of DNA vs the degree of confinement. The bulk values of the longest relaxation time $\tau_{1, \text { bulk }}$ and the radius of gyration $R_{\mathrm{g}, \text { bulk }}$ are estimated using $\tau_{1, \text { bulk }}=$ $0.1 \eta_{\mathrm{s}}\left(M / M_{\lambda-\mathrm{DNA}}\right)^{1.8} \mathrm{~s}$, and $R_{\mathrm{g}, \mathrm{bulk}}=0.7\left(M / M_{\lambda-\mathrm{DNA}}\right)^{0.6} \mu \mathrm{m}$. The dashed line is the regression over the results of $\lambda$-DNA in different channels from the current study. The black symbols indicate the data of this study. The hollow symbols indicate those from Randall and Doyle. ${ }^{21-23}$ The cross symbols are estimated from Bakajin et al. ${ }^{17}$ The diamond, round, up-triangle, down-triangle, and square symbols represent $1 / 2$ $\lambda$-DNA, $\lambda$-DNA, $2 \lambda$-DNA, T4-DNA, and BAC-DNA, respectively. Except for the current study, the longest relaxation times of DNA were measured by observing the relaxation of an initially stretched DNA.

and $D$ with $\mathrm{N}$ remains the same while those with $h$ change. Using $v_{3 \mathrm{~d}}=0.588$ leads to an even larger discrepancy between the experimental results and the blob theory predictions with Zimm-blob assumption. This finding reinforces our conclusion that blobs are partial-draining.

5.5. Comparison to Relaxation Times in Other Studies. The goal of this section is twofold. First, we want to compare the DNA relaxation times measured in the current study with those reported in other studies. Second, we want to construct a master plot of DNA relaxation time and thus provide an easy way for estimating $\tau_{1}$ of DNA molecules in different degrees of confinement.

If de Gennes' prediction is perfectly held, one expects $\left(\tau_{1} /\right.$ $\left.\tau_{1, \text { bulk }}\right) \sim\left(h / R_{\mathrm{g}, \text { bulk }}\right)^{-7 / 6}$, where $\left(\tau_{1} / \tau_{1 \text {,bulk }}\right)$ depends only on the degree of confinement, but is independent of the polymer molecular weight, $M$. Here $M$ is equivalent to $N$ defined in an earlier section. To reach the above formula, we have used $\tau_{1, \text { bulk }}$ $\sim M^{1.8}$ and $R_{\mathrm{g}, \text { bulk }} \sim M^{0.6} .{ }^{30,48}$ Since we observe deviation from de Gennes' prediction in our experiments, we use measured scalings and reach a similar formula: $\left(\tau_{1} / \tau_{1, \text { bulk }}\right) \sim M^{0.1}(h /$ $\left.R_{\mathrm{g}, \text { bulk }}\right)^{-0.92}$. Because of the very weak $M$ dependence, we are able to construct a master plot for comparison and estimation of the relaxation time of a DNA under different degrees of confinement. We collect the data from this study, Randall and Doyle, ${ }^{21,22}$ Randall, ${ }^{23}$ and Bakajin et al. ${ }^{17}$ and plot $\left(\tau_{1} / \tau_{1, \text { bulk }}\right)$ vs $\left(R_{\mathrm{g}, \text { bulk }} / h\right)$ in Figure 6. The bulk values of the longest relaxation time $\tau_{1, \text { bulk }}$ and the radius of gyration $R_{\mathrm{g} \text {,bulk }}$ are estimated using $\tau_{1, \mathrm{bulk}}=0.1 \eta_{\mathrm{s}}\left(M / M_{\lambda-\mathrm{DNA}}\right)^{1.8} \mathrm{~s}$, and $R_{\mathrm{g}, \mathrm{bulk}}=0.7\left(M / M_{\lambda-\mathrm{DNA}}\right)^{0.6}$ $\mu \mathrm{m} .{ }^{38,39,48} \eta_{\mathrm{s}}$ is the solvent viscosity in the unit of centipoise (cP). We note that the DNA relaxation time collected from other studies were all measured from the relaxation of initially stretched DNA. To estimate the relaxation times of T4-DNA in Bakajin et al., ${ }^{17}$ we have used

$$
\left(X^{2}-X_{0}^{2}\right)=A \exp \left(-t / \tau_{1}\right)
$$

where $X^{2}$ is the stretch square obtained from their Figure 4, $X_{0}^{2}$ is the equilibrium value of $X^{2}$, and $A$ is a free parameter. We find $\tau_{1} \approx 2.5,3.6$, and $6.8 \mathrm{~s}$ in $5 \mu \mathrm{m}, 300 \mathrm{~nm}$, and $90 \mathrm{~nm}$ slits, respectively. These values are in agreement with their qualitative description that the relaxation time of a T4-DNA was doubled when the slit height decreased from $5 \mu \mathrm{m}$ to $300 \mathrm{~nm}$ and was doubled again when $h$ decreased from 300 to $90 \mathrm{~nm}$.

As can be seen in Figure 6, all our data and those of Randall and Doyle fall along the dashed line within a narrow band for $R_{\mathrm{g}, \text { bulk }} / h>0.5$. The dashed line with the slope of 0.92 is the regression over measured $\tau_{1}$ of $\lambda$-DNA in different channels from the current study. $\tau_{1}$ of other sizes of DNA measured in $475 \mathrm{~nm}$ high channels also agree well with the regression. In order to further verify the master plot, we also measure the rotational relaxation time of T4-DNA in a $1 \mu \mathrm{m}$ slitlike glass channel (donated by U.S. Genomics). The result is shown as the black down-triangle in Figure 6, and it does follow the dashed line closely. The results of Randall and Doyle spread in the region where DNA is less confined. Their results start to deviate from the dashed line at $R_{\mathrm{g}, \mathrm{bulk}} / h \approx 0.5$, where the channel height is equal to the unconfined coil size of a DNA. Although the transition is very smooth, it indicates where blob theory starts to break down. On the other hand, the relaxation times estimated from Bakajin et al., although in qualitative agreement with our results, deviate significantly from the master curve. The cause of this discrepancy is not yet clear, but we suspect that it is because the data of Bakajin et al. are singlechain results. Because of molecular individualism, ${ }^{49}$ even physically identical molecules can behave differently under identical conditions. Therefore, accurate results are only accessible through analysis of an average of many single-chain results. Another possible cause of this discrepancy is that Bakajin's data only covered the normalized chain extension $\bar{X}$ from 0.6 to 0.2 while the true relaxation time is only accessible near equilibrium. $\bar{X}$ is defined as the ratio of DNA stretch to its contour length. In single-molecule studies, the relaxation time is typically fitted where $\bar{X}$ is smaller than 0.3 . We also note that one tends to obtain a relaxation time smaller than the true relaxation time when monitoring the relaxation of an initially highly stretched chain in good solvent. The reason is that although the excluded volume has strong effect on the relaxation time of a chain, it has little effect even when $\bar{X}$ is around 0.3 .

5.6. Effective Relaxation Time for Confined DNA. A polymer will be deformed from its equilibrium when Weissenberg number $W i=\tau_{\text {effective }} \dot{\gamma}$ is larger than one. Although $\tau_{\text {effective }}$ is typically taken as the longest relaxation time $\tau_{1}$ in bulk, this is not always true for a confined polymer. For a chain confined in a slit, if the flow gradient is imposed over the $x y$ plane $(z$ direction is the smallest dimension), $\tau_{\text {effective }}$ is indeed equal to $\tau_{1}$. As a result, long DNA will be stretched much easier than short DNA because $\tau_{1}$ scales as $N^{2.5}$. Such a flow condition can be generated, for instance, in a slit with a contraction in either $x$ or $y$ direction. ${ }^{50}$ On the other hand, if the flow gradient is applied along $z$ direction such as in simple shear flow or in Poiseuille flow, all confined chains, regardless their size, will start to deform from equilibrium at the same critical strain rate. This is because the flow gradient is only applied over the size of a blob where $\tau_{\text {blob }}$ is the governing time constant at this length scale, and $\tau_{\text {blob }}$ depends only on the channel height but not on DNA molecular weight. ${ }^{14}$

While we cannot measure $\tau_{\text {blob }}$ directly, we can estimate it given that $\tau_{1}$ is known. For instance, Balducci et al. ${ }^{27}$ have estimated the $R_{\mathrm{g}}$ of a M13mp18 DNA $(7.2 \mathrm{kbp})$ to be $240 \mathrm{~nm}$. Therefore, a blob in a $475 \mathrm{~nm}(\sim 2 \times 240 \mathrm{~nm})$ slit will contain 
roughly $7.2 \mathrm{kbp}$. Since we have shown blobs follow a 2D SAW, the relaxation time of a blob can be related to its longest relaxation time by $\tau_{1}=N_{\text {blob }}{ }^{2 v_{2 d}+1} \tau_{\text {blob. }}{ }^{24,32}$ Using $\tau_{1, \mathrm{BAC}-\mathrm{DNA}, 475 \mathrm{~nm}}$ $=11.1 \mathrm{~s}$ and the experimental scaling $2 v_{2 \mathrm{~d}}+1 \approx 2.48$, we obtain $\tau_{\text {blob, } 475 \mathrm{~nm}} \approx 3.5 \times 10^{-3} \mathrm{~s}$. Therefore, if one wants to deform a linear DNA confined in a $475 \mathrm{~nm}$ slit with a shear flow, a shear rate of at least $300 \mathrm{~s}^{-1}$ is required. We can roughly verify this value of $\tau_{\text {blob }}$ by comparing it with the relaxation time of a $7.2 \mathrm{kbp}$ DNA in bulk solutions. Using $\tau_{1, \lambda-\mathrm{DNA}}=0.1$ $\mathrm{s}$ in water with Zimm scaling in good solvent, we obtain $\tau_{1,7.2 \mathrm{kbpNA}} \approx 3.3 \times 10^{-3} \mathrm{~s}$ that is a little lower than the estimated $\tau_{\text {blob, } 475 \mathrm{~nm} .}$

Having the above idea in mind, we are able to explain some unusual observations reported in recent literature. ${ }^{8,50}$ For instance, Larson et al. ${ }^{50}$ characterized the average stretch of BAC-DNA $\left(R_{\mathrm{g} \text {,bulk }} \approx 1.6 \mu \mathrm{m}\right)$ confined in a $1 \mu \mathrm{m}$ glass slit in Poiseuille flow. A significant rise in BAC-DNA stretch was observed from a gap averaged shear rate $\gamma \approx 250 \mathrm{~s}^{-1}$, and the DNA stretch did not reach a plateau until $\dot{\gamma} \approx 6000 \mathrm{~s}^{-1}$. We estimate $\tau_{1} \approx 4 \mathrm{~s}$ and $\tau_{\text {blob }} \approx 0.04 \mathrm{~s}$. Therefore, if one naively

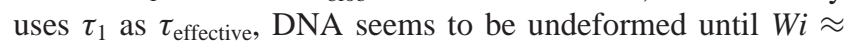
1000 , and its stretch does not saturate until $W i \approx 24000$ ! However, using $\tau_{\text {blob }}$ as $\tau_{\text {effective, }}$, we find that DNA starts to stretch at $W i \approx 10$ and its stretch saturates at $W i \approx 240$. It is not surprising that DNA did not start to deform at $W i \approx 1$ since the velocity gradient is nonuniform in Poiseuille flow. Similarly, Stein et al. ${ }^{8}$ measured the mobility of $\lambda$-DNA in Poiseuille flow in a $250 \mathrm{~nm}$ high slit (see their Figure 1). The gap averaged shear rate was around $90 \mathrm{~s}^{-1}$ while no flow-induced deformation of DNA was observed. Again, if $\tau_{1} \approx 0.7 \mathrm{~s}$ is used, $W i \approx 60$, while we estimate the true $W i=\tau_{\text {blob }} \dot{\gamma} \approx 0.06$. The same phenomenon has been predicted and discussed by Jendrejack et al. ${ }^{14,51}$ for DNA confined in square channels. Woo et al. ${ }^{20}$ also demonstrated in simulations that the tumbling dynamics of DNA is hindered in confinement, and the dominating time constant of the tumbling dynamics is much smaller than the longest relaxation time measured in the $x y$ plane.

\section{Conclusions}

In summary, we have measured the diffusivity and the longest relaxation time of DNA confined in siltlike nanochannels and find $\tau_{1} \sim M^{2.45} h^{-0.92}$. Comparing the experimental results with the predictions given by blob theory, we find that DNA blobs follow a 2D self-avoiding walk, and DNA segments within blobs follow a 3D self-avoiding walk. However, the assumption that hydrodynamic interactions dominate within blobs is found to be compromised. Our results indicate that blobs are partialdraining in the channels that we investigate. We expect that the assumption of nondraining blobs might become valid in the limit that a very long polymer confined in a large slit. In that case, there are enough polymer segments within a blob to induce very strong intersegment hydrodynamic interactions. Using the experimental scalings, we find $\left(\tau_{1} / \tau_{1, \text { bulk }}\right) \sim M^{0.1}\left(R_{\mathrm{g}, \text { bulk }} / h\right)^{0.92}$. The very weak $M$ dependence allows us to provide a master plot of $\left(\tau_{1} / \tau_{1, \text { bulk }}\right)$ vs $\left(R_{\mathrm{g}, \text { bulk }} / h\right)$. Relaxation times of confined DNA reported in other studies are collected in the master plot for comparison. Our results are in good agreement with those of Randall and Doyle, ${ }^{21-23}$ but not with those of Bakajin et al. ${ }^{17}$ We also addressed how the longest relaxation time and the blob relaxation time of DNA are related and when they are the dominant time constant in different flow conditions. We explain the recent observations that extreme high shear rate is required to deform a confined DNA using the blob picture.
Acknowledgment. The authors thank U.S. Genomics for providing the BAC-DNA samples and $1 \mu \mathrm{m}$ high slitlike glass channels. The authors also thank Professor Jongyoon Han and Mr. Pan Mao for instructions on manufacturing the nanoslits. This work was supported by U.S. Genomics and the National Science Foundation CAREER Grant CTS-0239012.

\section{References and Notes}

(1) Han, J.; Craighead, H. G. Science 2000, 288, 1026.

(2) Tegenfeldt, J. O.; Prinz, C.; Cao, H.; Chou, S.; Reisner, W.; Riehn, R.; Wang, Y. M.; Cox, E. C.; Sturm, J. C.; Silberzan, P.; Austin, R. H. Proc. Natl. Acad. Sci. U.S.A. 2004, 101, 10979.

(3) Riehn, R.; Lu, M.; Wang, Y.-M.; Lim, S. F.; Cox, E. C.; Austin, R H. Proc. Natl. Acad. Sci. U.S.A. 2005, 102, 10012.

(4) Reisner, W.; Morton, K. J.; Riehn, R.; Wang, Y. M.; Yu, Z.; Rosen, M.; Sturm, J. C.; Chou, S. Y.; Frey, E.; Austin, R. H. Phys. Rev. Lett. 2005, 94, 196101.

(5) Lagerqvist, J.; Zwolak, M.; Di Ventra, M. Nano Lett. 2006, 6, 779

(6) Fu, J.; Schoch, R. B.; Stevens, A. L.; Tannenbaum, R. S.; Han, J. Nat. Nanotechnol. 2007, 2, 121.

(7) Jo, K.; Dhingra, D. M.; Odijk, T.; de Pablo, J. J.; Graham, M. D.; Runnheim, R.; Forrest, D.; Schwartz, D. C. Proc. Natl. Acad. Sci. U.S.A. 2007, 104, 2673.

(8) Stein, D.; van der Heyden, F. H. J.; Koopmans, W. J. A.; Dekker, C. Proc. Natl. Acad. Sci. U.S.A. 2006, 103, 15853.

(9) Mao, P.; Han, J. Lab. Chip 2005, 5, 837.

(10) Brochard, F.; de Gennes, P. G. J. Chem. Phys. 1977, 67, 52.

(11) Daoud, M.; de Gennes, P. G. J. Phys. (Paris) 1977, 38, 85.

(12) Brochard, F. J. Phys. (Paris) 1977, 38, 1285.

(13) Odijk, T. Macromolecules 1983, 16, 1340.

(14) Jendrejack, R. M.; Dimalanta, E. T.; Schwartz, D. C.; Graham, M. D.; de Pablo, J. J, Phys. Rev. Lett. 2003, 91, 038102.

(15) Jendrejack, R. M.; Schwartz, D. C.; Graham, M. D.; de Pablo, J. J. J. Chem. Phys. 2003, 119, 1165.

(16) Odijk, T. J. Chem. Phys. 2006, 125, 204904.

(17) Bakajin, O. B.; Duke, T. A. J.; Chou, C. F.; Chan, S. S.; Austin, R. H.; Cox, E. C. Phys. Rev. Lett. 1998, 80, 2737.

(18) Stigter, D. Biophys. Chem. 2002, 101-102, 447.

(19) Woo, N. J.; Shaqfeh, E. S. G.; Khomami, B. J. Rheol. 2004,48, 281.

(20) Woo, N. J.; Shaqfeh, E. S. G.; Khomami, B. J. Rheol. 2004,48, 299.

(21) Randall, G. C.; Doyle, P. S. Macromolecules 2005, 38, 2140.

(22) Randall, G. C.; Doyle, P. S. Macromolecules 2006, 39, 7734

(23) Randall, G. C. Single Molecule Analysis of DNA Electrophoresis in Microdevices. Ph.D. Thesis, Massachusetts Institute of Technology, 2006.

(24) Hagita, K.; Koseki, S.; Takano, H. J. Phys. Soc. Jpn. 1999, 68, 2144.

(25) Chen, Y.-L.; Graham, M. D.; de Pablo, J. J.; Randall, G. C.; Gupta, M.; Doyle, P. S. Phys. Rev. E 2004, 70, 060901(R).

(26) Usta, O. B.; Ladd, A. J. C.; Butler, J. E. J. Chem. Phys. 2005, 122, 094902.

(27) Balducci, A.; Mao, P.; Han, J.; Doyle, P. S. Macromolecules 2006, $39,6273$.

(28) Larson, R. G. J. Rheol. 2005, 49, 1.

(29) Flory, P. J. Principles of Polymer Chemistry; Cornell University Press: Ithaca, NY, 1953.

(30) Doi, M.; Edwards, S. F. The Theory of Polymer Dynamics; Oxford University Press: New York, 1986.

(31) de Gennes, P. G. Macromolecules 1976, 9, 587.

(32) Rubinstein, M.; Colby, R. H. Polymer Physics; Oxford University Press: New York, 2003.

(33) Van Vilet, J. H.; Luyten, M. C.; ten Brinke, G. Macromolecules 1992 $25,3802$.

(34) de Gennes, P. G. Macromolecules 1976, 9, 594.

(35) Happel, J.; Brenner, H. Low Reynolds Number Hydrodynamics; Kluwer: Boston, 1983.

(36) Milchev, A.; Binder, K. J. Phys. II 1996, 6, 21.

(37) Randall, G. C.; Schultz, K. M.; Doyle, P. S. Lab. Chip 2006, 6, 516.

(38) Perkins, T. T.; Smith, D. E.; Chu, S. Science 1997, 276, 2016.

(39) Fang, L.; Hu, H.; Larson, R. G. J. Rheol. 2005, 49, 127.

(40) Adachi, K.; Kotaka, T. Prog. Polym. Sci. 1993, 18, 585.

(41) Perkins, T. T. Exploring Polymer Dynamics with Single DNA Molecules. Ph.D. Thesis, Stanford University, 1997.

(42) Wang, Y. C.; Stevens, A. L.; Han, J. Anal. Chem. 2005, 77, 4293.

(43) Savin, T.; Doyle, P. S. Biophys. J. 2005, 88, 623.

(44) Maier, B.; Rädler, J. O. Phys. Rev. Lett. 1999, 82, 1911

(45) Maier, B.; Rädler, J. O. Macromolecules 2000, 33, 7185.

(46) Haber, C.; Ruiz, S. A.; Wirtz, D. Proc. Natl. Acad. Sci. U.S.A. 2000, 97, 10792.

(47) Doi, M.; Nakajima, H. Chem. Phys. 1974, 6, 124.

(48) Smith, D. E.; Perkins, T. T.; Chu, S. Macromolecules 1996, 29, 1372. 
(49) de Gennes, P. G. Science 1997, 276, 199.

(50) Larson, J. W.; Yantz, G. R.; Zhong, Q.; Charnas, R.; DAntoni, C. M.; Gallo, M. V.; Gillis, K. A.; Neely, L. A.; Phillips, K. M.; Wong, G. G.; Gullans, S. R.; Gilmanshin, R. Lab. Chip 2006, 6, 1187.
(51) Jendrejack, R. M.; Schwartz, D. C.; de Pablo, J. J.; Graham, M. D. J. Chem. Phys. 2004, 120, 2513.

MA070570K 